This item was submitted to Loughborough's Research Repository by the author.

Items in Figshare are protected by copyright, with all rights reserved, unless otherwise indicated.

\title{
Physical mechanisms of inactivation of Bacillus subtilis spores using cold atmospheric plasmas
}

PLEASE CITE THE PUBLISHED VERSION

PUBLISHER

(c) IEEE

VERSION

VoR (Version of Record)

LICENCE

CC BY-NC-ND 4.0

\section{REPOSITORY RECORD}

Deng, Xu-Tao, J.J. Shi, and Michael G. Kong. 2019. "Physical Mechanisms of Inactivation of Bacillus Subtilis Spores Using Cold Atmospheric Plasmas". figshare. https://hdl.handle.net/2134/5239. 
This item was submitted to Loughborough's Institutional Repository (https://dspace.lboro.ac.uk/) by the author and is made available under the following Creative Commons Licence conditions.

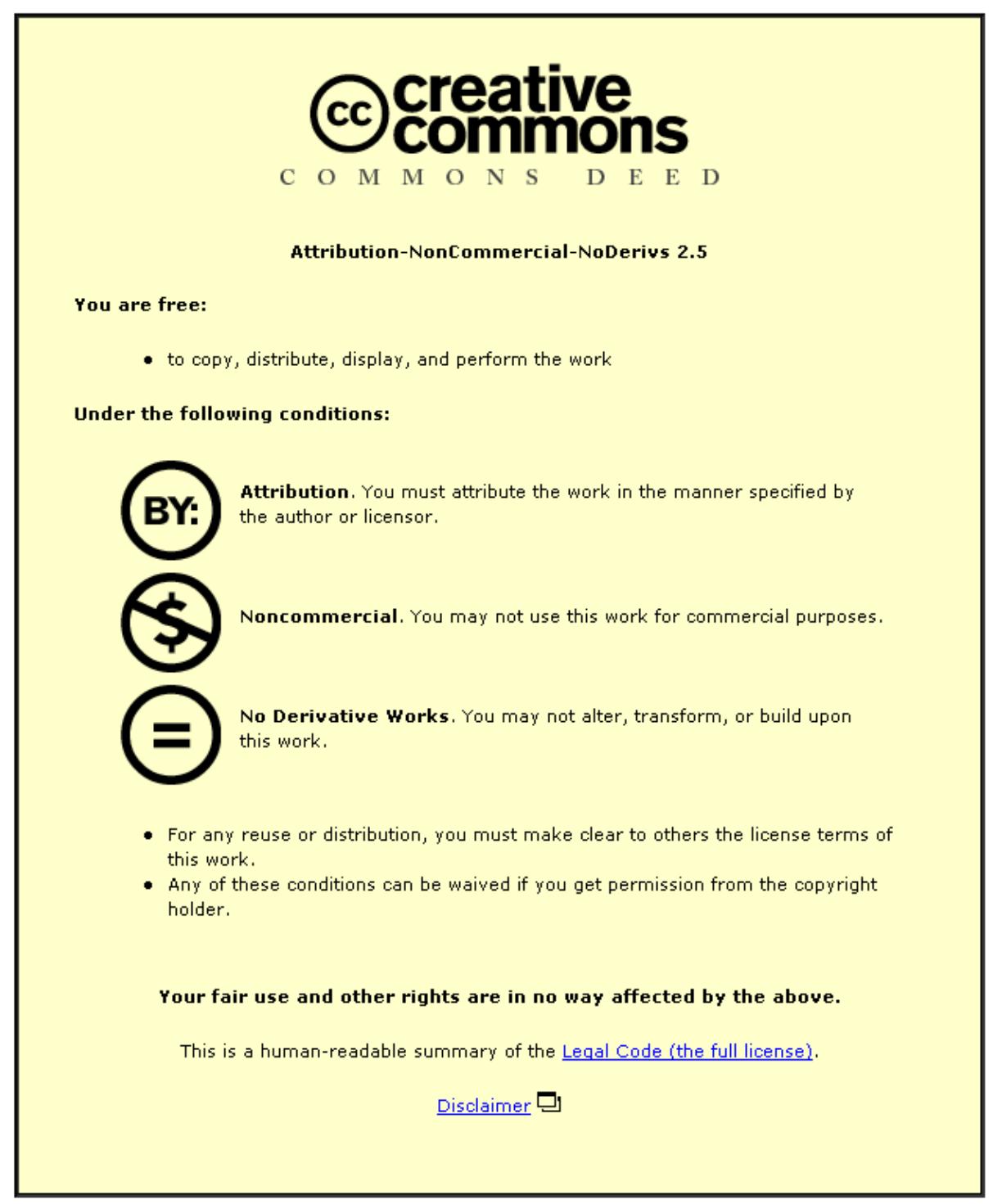

For the full text of this licence, please go to: http://creativecommons.org/licenses/by-nc-nd/2.5/ 


\title{
Physical Mechanisms of Inactivation of Bacillus subtilis Spores Using Cold Atmospheric Plasmas
}

\author{
Xutao Deng, Jianjun Shi, and Michael G. Kong, Senior Member, IEEE
}

\begin{abstract}
This paper presents a detailed study of the potential physical mechanisms of the microbial inactivation by cold atmospheric plasmas. With the Bacillus subtilis spores as a model microorganism and an atmospheric-plasma plume in helium flow, optical emission spectroscopy and inactivation kinetics are used to demonstrate the dominating role played by the reactive oxygen species (e.g., atomic oxygen and $\mathrm{OH}$ ) as well as the minor contributions of the UV photons, heat, charged particles, and electric fields. To differentiate the concentrations of the reactive oxygen species, an atmospheric helium-oxygen plasma is also used for the spore inactivation. Results with the helium and the helium-oxygen plasmas are contrasted to highlight how the production of the spore-killing oxygen species may be enhanced.
\end{abstract}

Index Terms-Atmospheric-pressure glow discharges (APGD), Bacillus subtilis spores, inactivation mechanisms.

\section{INTRODUCTION}

$\mathbf{T}$ HERE have been an increasing number of reports on the capability of the nonthermal plasmas to inactivate wide-ranging microorganisms [1], including Gram-negative and Gram-positive [1]-[6] as well as biofilm-forming bacteria [7]. Generated either in a vacuum chamber [2], [3] or in open air at atmospheric pressure [1], [4]-[7], the nonthermal gas discharges are now widely regarded as an exciting low-temperature decontamination technique. In spite of their demonstrated bactericidal capability, however, their inactivation mechanisms are largely unknown, thus preventing nonthermal plasmas from being rapidly developed into a mainstream decontamination technology.

In principle, plasma inactivation may be induced by the heat, charged particles, electric fields, UV photons, and reactive species (e.g., atomic oxygen, metastable oxygen molecules, ozone, and $\mathrm{OH}$ ); all are commonly present in a gas discharge. While these five physical quantities have long been linked to the microbial inactivation, such an association has so far been too general and nonspecific [2], [3], [5]. There are very few studies, if any, in the literature that have established their relative bactericidal capabilities. This is particularly so for the atmospheric plasmas of which different physical mechanisms may work together synergistically and so add a considerable complexity to their underpinning physics. On the other hand, microbiology-focused studies have recently been reported [5], [8]. They are concerned with the possible cellular sites, for

Manuscript received October 9, 2005; revised May 3, 2006. This work was supported in part by the Department of Health, U.K.

The authors are with Department of Electronic and Electrical Engineering, Loughborough University, LE11 3TU Leicestershire, U.K. (e-mail: m.g.kong@lboro.ac.uk).

Digital Object Identifier 10.1109/TPS.2006.877739 example peptidoglycan, which may have been hit by the plasma agents and become a vital trigger for bacterial death. Such studies are valuable, but currently, too few to substantiate and support a general understanding of the potential biological mechanisms of the plasma inactivation.

A full understanding of the plasma-inactivation mechanisms presents a major challenge at the interface between plasma physics and molecular microbiology. This is likely to require a validated identification of both the inactivation agents generated in the atmospheric plasmas and the damaged cellular sites in the bacterial structure. It is then necessary to correlate the bactericidal plasma agents to the plasma-hit cellular sites in the microorganism (e.g., cell membrane, peptidoglycan, cell-wall proteins and acids, DNA, or subcellular organelles). Clearly, this demands a systematic scientific program by many research groups over many years. The current study is a first attempt at the potential physical mechanisms of the atmospheric-plasma inactivation.

The motivation of this paper is to differentiate the roles in the microbial inactivation of the heat, charged particles, electric fields, UV photons, and reactive species. By using the Bacillus subtilis spores as a model microorganism and a cold atmospheric-plasma plume, we first present the key properties of the plasma-treated Gram-positive bacterium, including the leakage of the cytoplasm content and a complete membrane fragmentation. Then, we discuss the relative roles of the plasma-inactivation agents and demonstrate that a group of reactive plasma species was, to a very large extent, responsible for the observed spore inactivation. By contrast, all the other plasma agents (e.g., heat, charged particles, UV photons, and electric fields) played a minor role. Finally, we report results of a complimentary study aimed to enhance the spore inactivation by increasing the oxygen dissociation.

\section{Experimental Details}

\section{A. Bacterium and Cultivation}

The strain of the Bacillus subtilis used in this paper was ATCC 6633/NCIMB 8054 and was sporulated following the method of Harnulv and Snygg [9]. After a six-day incubation period at $30^{\circ} \mathrm{C}$, the resulting spores were harvested by centrifugation at $7367 \times \mathrm{g}$ at $5{ }^{\circ} \mathrm{C}$ for $20 \mathrm{~min}$. They were then washed four times in order to remove all the unwanted constituents. To ensure that the spore samples were free from any vegetative cells, a heat shock was applied by leaving the suspension in a $70{ }^{\circ} \mathrm{C}$ water bath for $30 \mathrm{~min}$. The spore suspensions were refrigerated until required. 


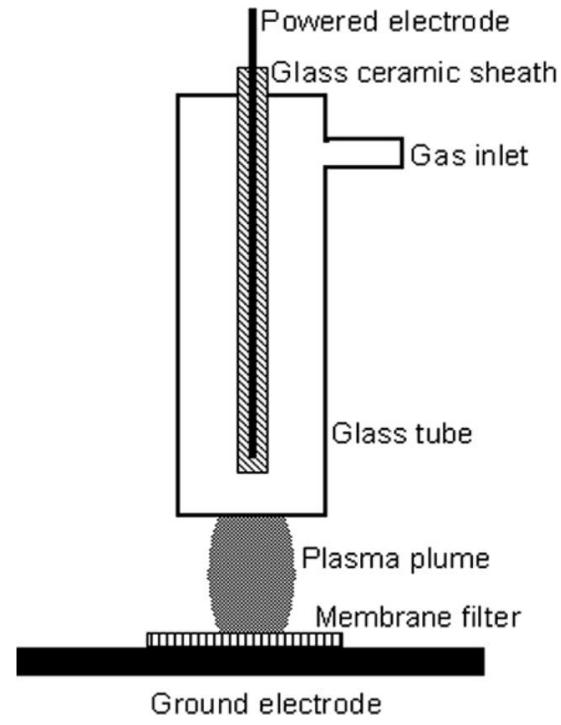

Fig. 1. Schematic of the cold atmospheric-plasma apparatus.

For convenience and ease of reproducibility, the spores were deposited onto the surface of the Whatman polycarbonate membranes (Fisher), which were supported by a layer of technical no. 3 agar in a petri dish. The spore suspension was diluted to the required concentration at approximately $10^{7}$ spores $/ \mathrm{ml}$, for the studies reported here, and $100 \mu \mathrm{l}$ of the solution was pipetted onto every membrane filter. The sporeladen membranes were then left to dry in a laminar flow cabinet before the plasma treatment. After the plasma treatment, the treated spores were recovered from the ringer solution (Oxoid) from the membranes. Serial dilutions were applied to all the recovered solution, after which the diluted solution were plated onto the Tryptone Soya Agar (Oxoid) plates and incubated at $30{ }^{\circ} \mathrm{C}$ overnight. Viable counts are expressed in terms of colony-forming units (CFU) per filter.

Microbial-loading levels have recently been shown to affect the plasma-inactivation efficacy [8]. To ensure that all the plasma-inactivation experiments reported here had a comparable microbial loading, the initial bacterial densities on the membrane filters were controlled to be within a narrow range of 3.5-7 $\times 10^{6}$ spores/filter or $4.5-9.0 \times 10^{6}$ spores $/ \mathrm{cm}^{2}$ with a spore-laden area of $0.78 \mathrm{~cm}^{2}$ on the membrane filter. Within this range of the initial spore density, the plasma-inactivation efficacy has been shown to change a little [8], and therefore, the microbial-loading effect was kept the same for all the results presented here.

\section{B. Cold Atmospheric-Plasma Plume System}

The plasma system used in this paper was the same as that used previously [8]. As shown in Fig. 1, it had a coaxial configuration with a powered copper electrode rod and a glass ceramic sheath. A concentric glass tube enclosing the electrode unit was added to minimize the environmental interference. Therefore, it was effectively a dielectric-barrier configuration either with a ground electrode at the sample holder or with the glass tube as a floating second electrode. For the data presented in this paper, the floating-electrode arrangement was used. The working gas was fed through the space between the powered electrode and the glass tube for ionization. The ionized gas was then flushed out of the electrode unit and into the ambient air toward a downstream point, about $1 \mathrm{~cm}$ away from the plasma-generation region, where the spore-containing membrane filter was placed and plasma treated. Pure helium or a helium-oxygen mixture was chosen as the background gas for the gas ionization. The helium flow was fixed at $3 \mathrm{slm}$, and the oxygen flow rate varied from 0 to $2 \mathrm{sccm}$, representing a maximum oxygen content of $0.07 \%$ in the background gas. Higher oxygen content was found to quench the plasma due to oxygen being an electronegative gas, although this can be overcome with a larger excitation voltage. A homemade power supply provided a high voltage to the plasma rig typically at $29-37 \mathrm{kHz}$ and $3-15 \mathrm{kV}$. This voltage level was sufficient to ignite both the pure helium and helium-oxygen mixture. For the pure helium flow, the plasma ignition occurred at a peak excitation voltage of $3 \mathrm{kV}$. With a greater applied voltage, the atmospheric-plasma plume appeared brighter until the peak voltage reached $7 \mathrm{kV}$, at which arcing was induced. For the helium-oxygen flow with the oxygen at $2 \mathrm{sccm}$, on the other hand, arcing was observed at a higher voltage of $12 \mathrm{kV}$.

Although the nonthermal atmospheric plasma was of relatively small size, it was appropriate, as our purpose was to enable a fundamental understanding rather than to cater for applications that demand technology upscaling. It is worth mentioning that the plasma inactivation of the microorganisms is feasible at scales larger than what is reported here [10].

\section{Measurement and Estimates of Plasma Parameters}

Electrical properties of the atmospheric-plasma plume were monitored using a Tektronics oscilloscope (TDS 3034B). The gas temperature near the sample was determined by comparing the measured and simulated optical emission of $\mathrm{OH}$ in a narrow spectral band between 306 and $310 \mathrm{~nm}$, based on a technique reported elsewhere [11]. Optical emission was measured using an Andor DH720/Shamrock system at a grating of $2400 \mathrm{~g} / \mathrm{mm}$. Effects of the UV photons were determined microbiologically by covering the bacterial sample with a UV fused silicon lens (ThorLabs, LA4380), which allowed light transmission at wavelengths as low as $185 \mathrm{~nm}$, but blocked all the charged and neutral plasma species. The cold atmosphericplasma plume used in this paper belonged to the class of atmospheric-pressure glow discharges (APGD) in which the electron density and the electric field are, at present, difficult to measure experimentally. As a result, the numerical range of these quantities was estimated from the computational studies [12].

\section{Physical Mechanisms of Plasma Inactivation}

The plasma inactivation of the microorganisms can be caused by five possible physical mechanisms namely: 1) heat; 2) charged particles; 3) electric field; 4) UV photons; and 5) reactive species. To differentiate their individual roles, a series of inactivation experiments was undertaken using the atmospheric-helium-plasma plume in Fig. 1. 

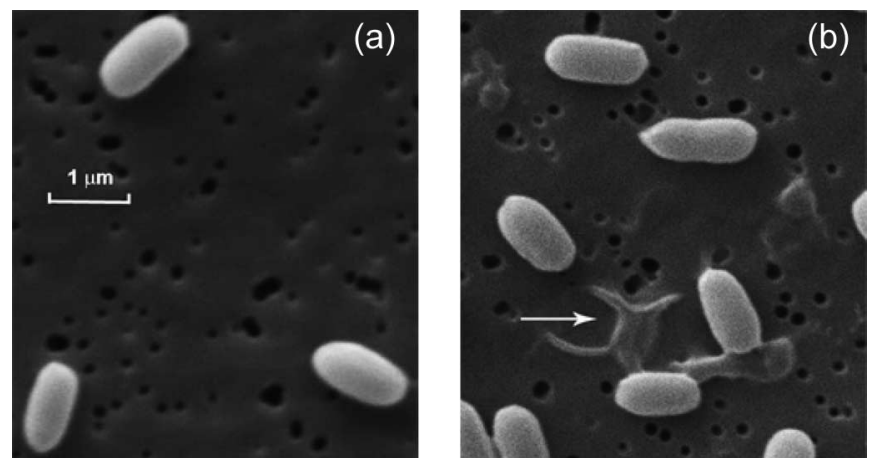

Fig. 2. SEM images of the Bacillus subtilis spores (a) before plasma treatment and (b) after plasma treatment for $5 \mathrm{~min}$ with a ruptured spore pointed.
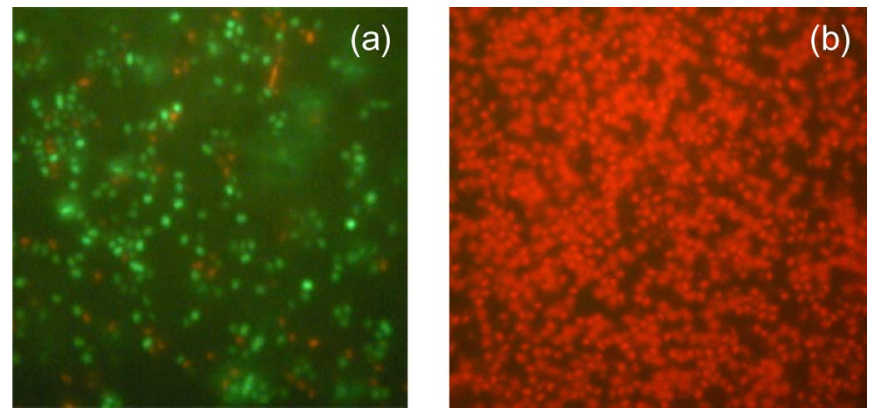

Fig. 3. Fluorescence images of the propidium iodide stained Bacillus subtilis spores (a) before plasma treatment and (b) after plasma treatment for $5 \mathrm{~min}$. (Color version available online at http://ieeexplore.ieee.org.)

\section{A. Plasma Damage of the Bacillus Subtilis Spores}

Morphological changes of the Bacillus subtilis spores after a 5-min treatment of the cold atmospheric plasma in pure helium flow are shown in the scanning electron microscope (SEM) images in Fig. 2. Evident were the plasma damages ranging from a slight shrinkage in size, through the leakage of the cytoplasm contents, to a complete rupture of the spore membrane (marked with an arrow in Fig. 2). In the past, substantial membrane rupture had been observed using similar SEM imaging techniques among the plasma-treated Gram-negative bacteria, but very rarely, if ever, for plasma-treated Gram-positive bacteria [1]-[7], [10]. The clear membrane rupture of some of the Bacillus subtilis spores in Fig. 2 was therefore exceptional. In addition, we stained the Bacillus Subtilis samples with propidium iodide, which allowed viable bacteria to fluoresce green and fatally injured bacteria to fluoresce red. Fluorescence images of the stained Bacillus subtilis samples before and after the plasma treatment, as shown in Fig. 3, clearly indicated a severe spore damage. The substantial spore damage demonstrated with the SEM and fluorescence microscope images in Figs. 2 and 3 was particularly intriguing since they were achieved using a pure helium atmospheric plasma, in which the reactive oxygen species were not expected to be abundant within the plasma. However, since the ionized helium was flushed into the ambient air, its electrons and ions could, in principle, impact on the spore sample. Impurity gases such as oxygen and nitrogen were inevitably present in the background helium gas, as our plasma rig was not enclosed in an air-tight chamber and so may assist UV generation. More importantly, in their passage from the

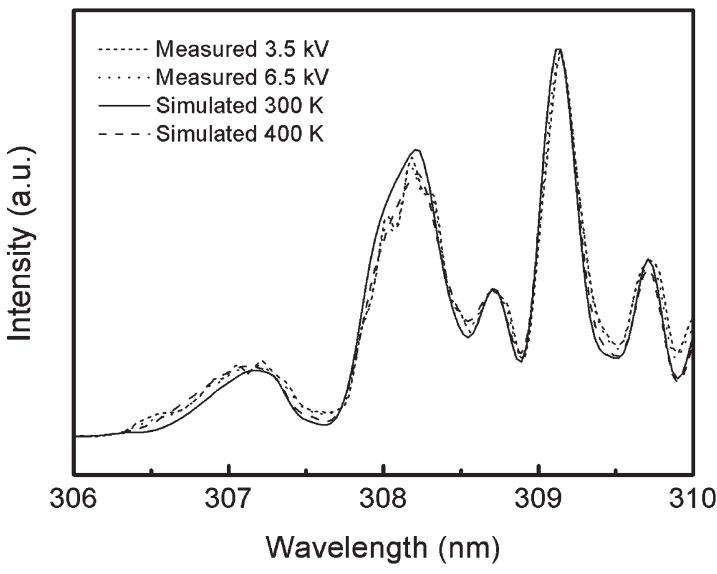

Fig. 4. Rotational temperature of $\mathrm{OH}$ in the helium atmospheric-plasma plume determined by comparing the measured and simulated emission spectrum.

plasma-generation region to the sample, the charged particles and the excited helium species could pass their kinetic energy to the ground-state oxygen molecules present in the ambient air. This could, in principle, create oxygen atoms, metastable oxygen molecules, ozone, and $\mathrm{OH}$, all of which are bactericidal.

\section{B. Effects of the Heat, Charged Particles, and Electric Field}

Rotational temperature of the $\mathrm{OH}$ in the atmospheric-heliumplasma plume exiting from the electrode unit was measured by means of optical emission spectroscopy [11], [13]. The results are shown in Fig. 4 in two different levels of the applied voltage, namely 3.5 and $6.5 \mathrm{kV}$, representing the lower and upper limits for a stable discharge generation in our plasma rig. The temperature range indicated in Fig. 3 was therefore the full range of the atmospheric-helium plasma plume. Fig. 4 suggests that the plasma temperature was between 300 and $350 \mathrm{~K}$ or below $77^{\circ} \mathrm{C}$. The actual temperature experience of the spore sample was much lower since the agar substrate would normally begin to melt at $47{ }^{\circ} \mathrm{C}$ and it did not show any sign of melting during any plasma treatment. So, our atmosphericplasma plume was near room temperature. As the Bacillus subtilis spores cannot be thermally inactivated below $80{ }^{\circ} \mathrm{C}$ [14], the plasma damages in Figs. 2 and 3 were almost certainly nonthermal.

The plasma treatment in our experiments was performed remotely at a downstream point approximately $1 \mathrm{~cm}$ away from the plasma-generation region, over which the ionized gas was estimated to have taken about 7-8 ms to reach the sample. By using the numerical models for the dielectric-barrier discharges in the atmospheric helium [15], [16], the recombination of the free electrons with the helium ions was found numerically to reduce the electron density by one magnitude over a period of 2-20 ms. This was consistent with the relevant experimental data [17]. Therefore, the electrons and the helium ions originally generated between the two electrodes could reach the spore sample and may have contributed significantly to the observed plasma damage in Fig. 2. To confirm their roles more clearly, comparison studies were performed with the spore sample placed either at the downstream point using 
our plasma plume (remote treatment) or inside a comparable parallel-plate dielectric-barrier discharge (in situ treatment), both in an atmospheric-helium flow. The spore inactivation (not shown) was found to be much more ineffective in the case of the in situ treatment, in which the electrons and the helium ions are most abundant. This suggests that the electrons and the helium ions were not responsible directly for the observed spore inactivation. It is however possible that the passage of the electrons and the helium ions from the electrode unit to the sample may have reacted with the ground-state oxygen molecules in the ambient air. As a result, this could produce abundant reactive oxygen species nearer or at the downstream sample. This issue will be revisited in Section III-D.

The remote mode of the plasma treatment implied no or little electric field at the location of the spore sample, and so, the electric field was unlikely to have contributed to the observed plasma damage in Fig. 2. For cases of in situ inactivation where the microbial samples were immersed within the plasmageneration region, the electric field could be significant. The maximum electric field in the nonthermal atmospheric glow discharges in helium is known to range from a few kilovolts per centimeter in the radio-frequency APGD [12], [18], through $20-30 \mathrm{kV} / \mathrm{cm}$ in the atmospheric dielectric-barrier glow discharges [16], to over $50 \mathrm{kV} / \mathrm{cm}$ in the dc APGD [19]. Such high electric fields could potentially breach the cell membrane [20]. However, for the atmospheric plasmas, they are unlikely to contribute significantly to the microbial inactivation. This is because the maximum electric field usually occurs at the cathode surface and then reduces exponentially to a fraction of its peak value over a very short distance of less than $300 \mu \mathrm{m}$ [16], [18]. Clearly, it was difficult to position the membrane well within $300 \mu \mathrm{m}$ from the cathode, and so, the electric field was unlikely to be a major factor in most plasma-inactivation experiments. This minor role was observed in an independent plasma-inactivation study [5].

\section{Effects of the UV Photons}

As the reference experiment, the Bacillus subtilis spores were exposed to the atmospheric-helium plasma plume over different durations so as to obtain the reference inactivation kinetics. In this case, the atmospheric plasma was sustained with a peak excitation voltage of $6.5 \mathrm{kV}$. To establish whether the UV photons may have played a major role, we placed the UV-passing glass above the membrane filter so that the spore sample was exposed to the UV photons only. The resulting inactivation kinetics were shown in Fig. 5 together. Control tests were performed with the spore samples exposed to a 3 -slm unionized helium flow for $10 \mathrm{~min}$. No reduction in the spore CFU count was found (not shown). From Fig. 5, it was clear that a 10-min exposure to the atmospheric-helium plasma plume led to a 4-log reduction of the Bacillus subtilis spores, whereas the use of the UV-passing glass resulted in a much reduced kill of less than 0.2-log reduction. It was therefore evident that the UV photons made a relatively minor contribution to the spore damage seen in Fig. 2. This was consistent with findings from similar atmospheric-plasma-inactivation studies [21]. It is worth emphasizing that UV generation can be sub-

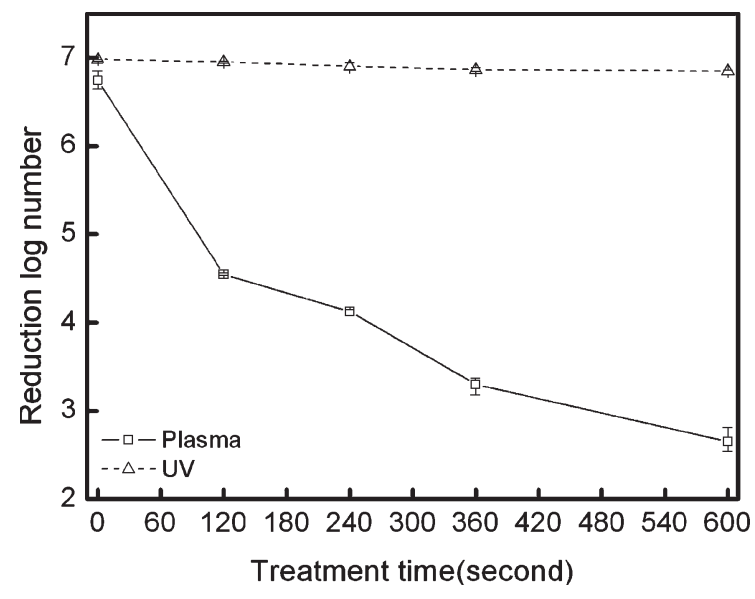

Fig. 5. Spore inactivation kinetics by an atmospheric-helium plasma plume (squares) and by the UV photons of this plasma alone (triangles).

stantially increased with the different background gases, and as such, UV photons could potentially play an important role. This is however outside the scope of the current study.

\section{Effects of the Reactive Oxygen Species}

To establish the roles of the reactive species, optical emission spectroscopy measurements were performed over a wide wavelength range from 200 to $800 \mathrm{~nm}$. For the wavelengths below $280 \mathrm{~nm}$, very little emission was detected, confirming that the UV photons were very few in the atmospheric plasma plume. The optical emission spectrum shown in Fig. 6 suggests that there were abundant $\mathrm{OH}$ molecules $(\sim 309 \mathrm{~nm})$, singlet-sigma-oxygen-metastables $(\sim 760 \mathrm{~nm})$ oxygen atoms $(\sim 777 \mathrm{~nm})$, and nitrogen ions. Singlet delta oxygen metastables were also observed, although their optical emission line of around $1270 \mathrm{~nm}$ was not included in Fig. 6. Ideally, it is preferable to measure the absolute concentrations of these reactive species and then to correlate their densities with the inactivation efficacy. This is however not always possible from their optical emission spectrum, and absolute density measurements are indeed a major research topic that requires a substantial future work. Nevertheless, the emission spectrum in Fig. 6 was indicative of the types of the reactive plasma species that may have been responsible for the achieved plasma inactivation of the Bacillus subtilis spores. It appears that the plasma species responsible for the spore inactivation in Figs. 2, 3 , and 5 included a large group of reactive plasma species such as oxygen atoms, metastable oxygen molecules, $\mathrm{OH}$, and several nitrogen-containing species. A low level of the ozone was also present in our experiments.

The results presented above have shown clearly that the observed spore damage in Fig. 2 was caused largely by the reactive plasma species, whereas the heat, charged particles, UV photons, and electric fields all played a minor role. This differentiation of the roles of the five physical quantities in the plasma inactivation should add a valuable contribution to the currently nonspecific understanding of their relative bactericidal abilities. The next logic step is to differentiate the individual roles of each reactive species in the microbial inactivation. This 

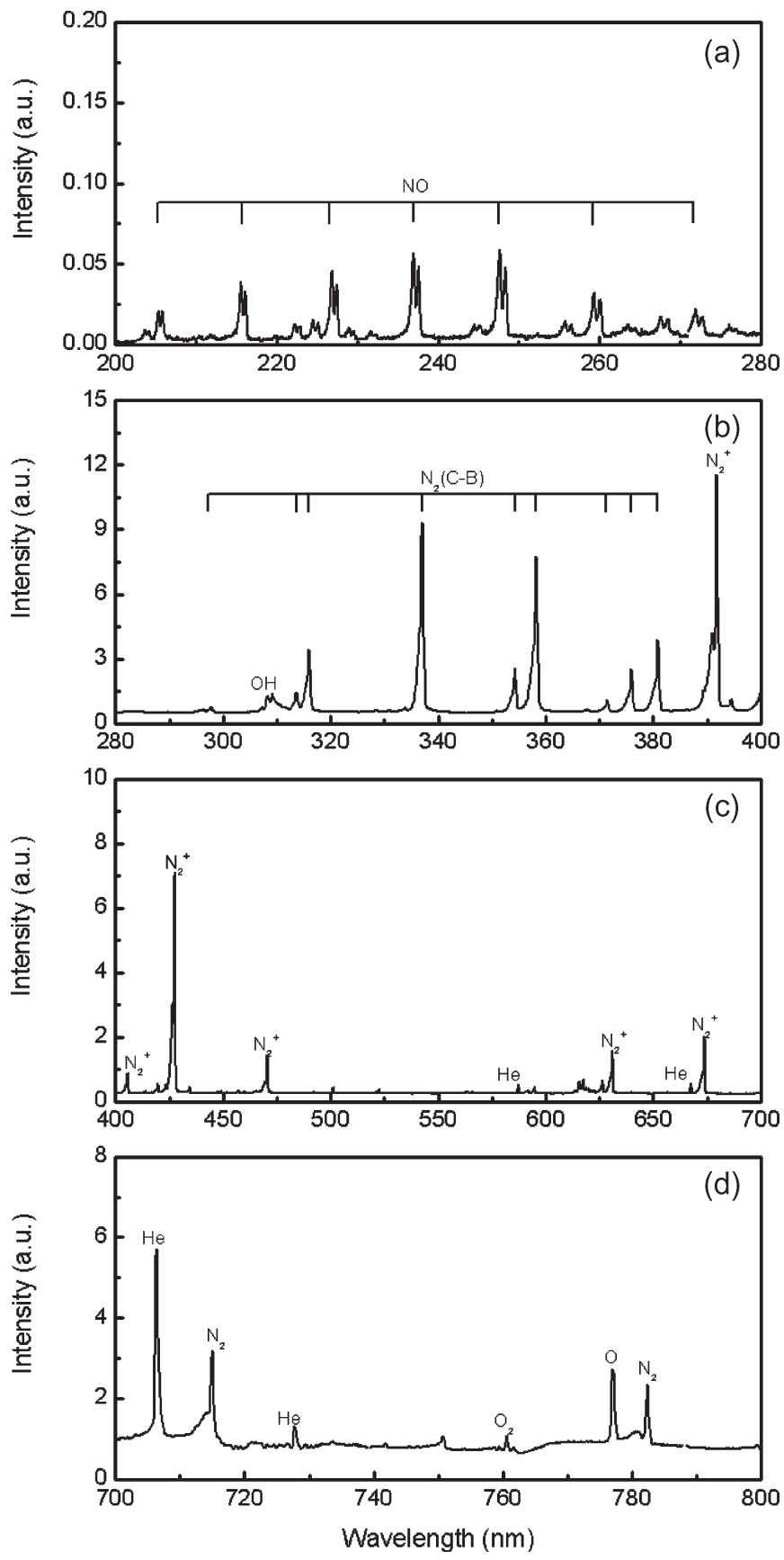

Fig. 6. Optical emission spectroscopy of the atmospheric-helium plume with the peak plasma-sustaining voltage at $6.5 \mathrm{kV}$.

is beyond the scope of the present paper and will be reported in a future note.

\section{Production of More Reactive Oxygen Species}

It has been established from the above discussion that the reactive oxygen species were likely to be the most important group of the inactivation agents in the cold atmospheric-plasma plume. This suggestion can be further supported if an enhanced production of these reactive oxygen species can be shown to result in a more effective spore inactivation. While offering an indirect evidence, it is an appropriate strategy of the investigation, given that the absolute density measurements of the

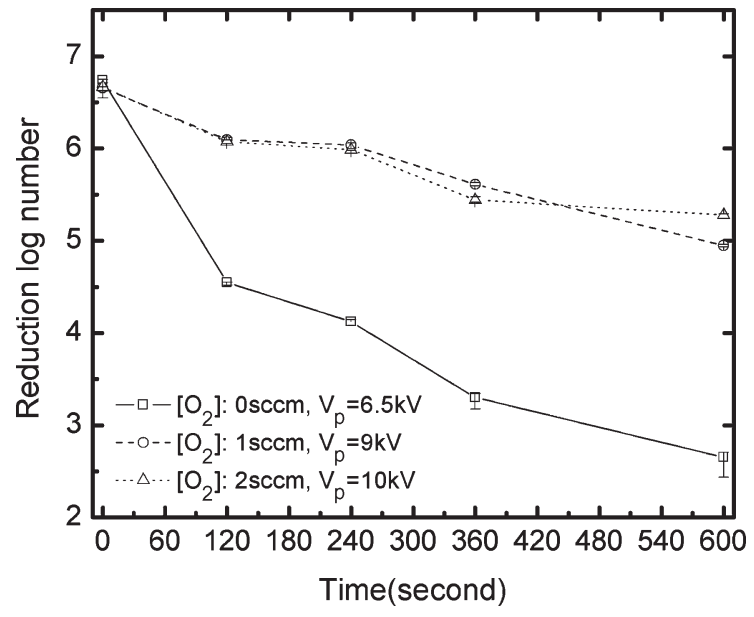

Fig. 7. Inactivation kinetics of the Bacillus subtilis spores by an atmospherichelium-oxygen plasma plume.

reactive oxygen species are not always straightforward. One obvious way to increase the production of the reactive oxygen species is to add a small amount of oxygen in the background helium flow [3], [22]. To this end, our coaxial electrode unit was used again to produce an atmospheric-helium-oxygen plasma plume, in which it was found necessary to increase the excitation voltage due to the high breakdown voltage of oxygen. The rotational temperature of $\mathrm{OH}$ in the helium-oxygen plume was found to remain below $350 \mathrm{~K}$, and again, no agar substrate melting was observed, suggesting that the sample temperature was below $47^{\circ} \mathrm{C}$. Therefore, the atmospheric-plasma treatment of the Bacillus subtilis spores was still nonthermal.

Inactivation kinetics are shown in Fig. 7 at three oxygen flow rates at 0,1 , and $2 \mathrm{sccm}$. The surprising result was that the introduction of a small amount of oxygen in the helium flow actually reduced the efficacy of the plasma inactivation. For a plasma exposure time of $10 \mathrm{~min}$, the $\log$ reduction of the Bacillus subtilis spores became more than half from 4.0 to 1.5. It was clear that the addition of oxygen in the background gas for the plasma generation did not translate into the more reactive oxygen species in the downstream near the sample point. This may be caused by the reduced level of gas ionization and oxygen dissociation in the helium-oxygen plasma, in which the addition of oxygen increased the breakdown voltage and introduced an additional electron-loss channel through the electron attachment to the oxygen gas.

We considered an alternative route to enhance the production of the reactive oxygen species in the atmospheric-plasma plume. This was to elevate the excitation voltage so that both the electron energy and the electron density were increased in the plasma-generation region near the sample. The passage of the ionized-helium flow with more energetic and more abundant electrons should in turn help increase the production of the reactive oxygen species in the downstream region. To confirm this, the original atmospheric-helium plume was used to inactivate the Bacillus subtilis spores at different levels of the excitation voltage, as shown in Fig. 8. As the peak voltage was increased from 3.5 to $6.5 \mathrm{kV}$, a 10 -min exposure to the atmospherichelium plume was seen to have caused the log reduction of the Bacillus subtilis spores to increase from 1.8 to 4.0. It was 


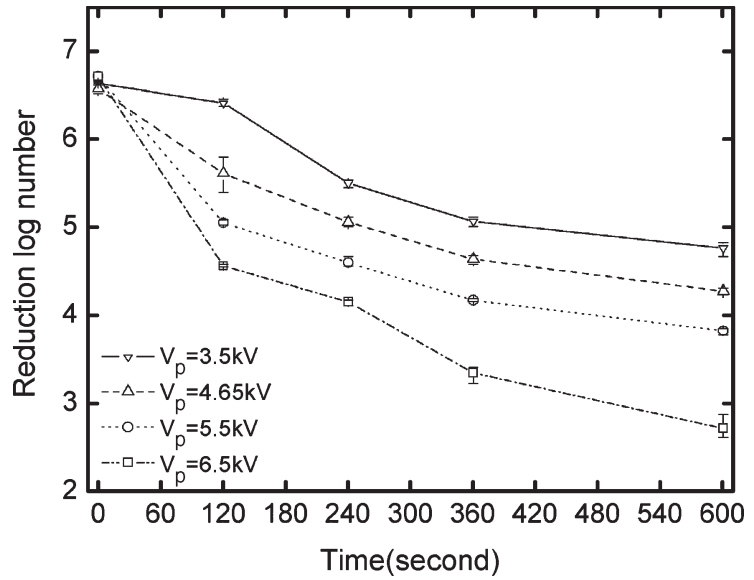

Fig. 8. Inactivation kinetics of the Bacillus subtilis spores by the atmospherichelium plasma plume at different ionization voltages.

therefore evident that an efficient production of the reactive oxygen species and, hence, an effective spore inactivation can be better facilitated with the atmospheric-helium plasma plume than with the atmospheric-helium-oxygen plasma plume.

\section{CONCLUSION}

This paper investigated the possible physical mechanisms in the Bacillus subtilis-spore inactivation by the nonthermal atmospheric-plasma plumes. The leakage of cytoplasm contents and a complete rupture of the spore membrane were observed. Through optical emission spectroscopy and carefully varied inactivation kinetics studies, it has been established that the observed spore inactivation was most probably induced by the reactive oxygen species with the heat, UV photons, electric field, and charged particles; all are making minor contributions. To improve the production of these reactive oxygen species and, hence, enhance spore inactivation, it was found that it is more effective to use an atmospheric-helium plasma plume rather than a comparable atmospheric-helium-oxygen plasma plume because the former supported a greater level of gas ionization and oxygen dissociation.

\section{ACKNOWLEDGMENT}

The authors would like to thank G. Shama (Department of Chemical Engineering, Loughborough University, U.K.) for the technical advice.

\section{REFERENCES}

[1] K. Kelley-Wintenberg, T. C. Montie, C. Brickman, J. R. Roth, A. K. Carr, K. Sorge, L. C. Wadsworth, and P. P. Y. Tsai, "Room temperature sterilization of surfaces and fabrics with a one atmosphere uniform glow discharge plasma," J. Ind. Microbiol. Biotechnol., vol. 20, no. 1, pp. 69-74, Jan. 1998.

[2] S. Moreau, M. Moisan, M. Tabrizian, J. Barbeau, J. Pelletier, A. Ricard, and L'H. Yahia, "Using the flowing afterglow of a plasma to inactivate Bacillus subtilis spores: Influence of the operating conditions," J. Appl. Phys., vol. 88, no. 2, pp. 1166-1174, Jul. 2000.

[3] N. Philip, B. Saoudi, M. Crevier, M. Moisan, J. Barbeau, and J. Pelletier, "The respective roles of UV photons and oxygen atoms in plasma sterilization at reduced gas pressure: The case of $\mathrm{N}_{2}-\mathrm{O}_{2}$ mixtures," IEEE Trans. Plasma Sci., vol. 30, no. 4, pp. 1429-1436, Aug. 2002.

[4] H. W. Hermann, G. S. Selwyn, I. Henins, J. Park, M. Jeffery, and J. M. Williams, "Chemical warfare agent decontamination studies in the plasma decon chamber," IEEE Trans. Plasma Sci., vol. 30, no. 4, pp. 1460-1470, Aug. 2002.

[5] J. G. Birmingham, "Mechanisms of bacterial spore deactivation using ambient pressure nonthermal discharges," IEEE Trans. Plasma Sci., vol. 32, no. 4, pp. 1526-1531, Aug. 2004.

[6] M. Laroussi, "Low temperature plasma-based sterilization: Overview and state-of-the-art," Plasma Proc. Polymers, vol. 5, no. 2, pp. 391-400, Jun. 2005.

[7] M. Vleugels, G. Shama, X. Deng, E. Greenacre, T. Brocklehurst, and M. G. Kong, "Atmospheric plasma inactivation of biofilm-forming bacteria for food safety control," IEEE Trans. Plasma Sci., vol. 33, no. 2, pp. 824-828, Apr. 2005.

[8] X. Deng, J. Shi, G. Shama, and M. Kong, "Effect of microbial loading and sporulation temperature on atmospheric plasma inactivation of Bacillus subtilis spores," Appl. Phys. Lett., vol. 87, no. 15, p. 153 901, Oct. 2005.

[9] B. G. Harnulv and B. G. Snygg, "Heat resistance of Bacillus subtilis spores at various water activities," J. Appl. Bacteriol., vol. 35, no. 4, pp. 615-624, Dec. 1972.

[10] A. Sharma, A. Pruden, Z. Q. Yu, and G. J. Collins, "Bacterial inactivation in open air by the afterglow plume emitted from a grounded hollow slot electrode," Environ. Sci. Technol., vol. 39, no. 1, pp. 339-344, Jan. 2005.

[11] S. Moon and W. Choe, "A comparative study of rotational temperature using diatomic $\mathrm{OH}, \mathrm{O}_{2}$ and $\mathrm{N}_{2}^{+}$molecular spectra emitted from atmospheric plasmas," Spectrochim. Acta, Part B: Atom. Spectrosc., vol. 58, no. 2, pp. 249-257, Feb. 2003.

[12] J. J. Shi and M. G. Kong, "Mode characteristics of radio-frequency atmospheric glow discharges," IEEE Trans. Plasma Sci., vol. 33, no. 2, pp. 624-630, Apr. 2005.

[13] J. H. Kim, Y. H. Kim, Y. H. Choi, W. Choe, J. J. Choi, and Y. S. Hwang, "Optical measurements of gas temperatures in atmospheric pressure RF cold plasmas," Surf. Coat. Technol., vol. 171, no. 1-3, pp. 211-215, Jul. 2003.

[14] W. L. Nicholson, N. Munakata, G. Horneck, H. J. Melosh, and P. Setlow, "Resistance of Bacillus endospores to extreme terrestrial and extraterrestrial environments," Microbiol. Mol. Biol. Rev., vol. 64, no. 3, pp. 548-572, Sep. 2000.

[15] M. G. Kong and X. T. Deng, "Electrically efficient production of a diffuse nonthermal atmospheric plasma," IEEE Trans. Plasma Sci., vol. 31, no. 4, pp. 7-18, Aug. 2003

[16] X. M. Zhu and M. G. Kong, "Electron kinetic effects in atmospheric dielectric-barrier glow discharges," J. Appl. Phys., vol. 97, no. 8, p. 083301 , Aug. 2005.

[17] O. Bilyk, M. Holik, A. Pysanenko, J. Glosik, P. Kudrina, R. Plasil, and M. Tichy, "Langmuir probe diagnostics for measurement of recombination rates of positive ions with electrons in stationary afterglow system," Vacuum, vol. 76, no. 4, pp. 457-463, Nov. 2004.

[18] J. J. Shi and M. G. Kong, "Evolution of discharge structure in capacitive radio-frequency atmospheric microplasmas," Phys. Rev. Lett., vol. 96, no. 10, p. 105 009, Mar. 2006.

[19] _ - "Cathode fall characteristics of a dc atmospheric glow discharge," J. Appl. Phys., vol. 94, no. 9, pp. 5504-5513, Oct. 2003.

[20] J. R. Beveridge, K. Wall, S. J. Macgregor, J. G. Anderson, and N. J. Rowan, "Pulsed electric field inactivation of spoilage microorganisms in alcoholic beverages," Proc. IEEE, vol. 92, no. 7, pp. 1138-1143, Jul. 2004.

[21] M. Yamamoto, M. Nishioka, and M. Sadakata, "Sterilization by $\mathrm{H}_{2} \mathrm{O}_{2}$ droplets under corona discharge," J. Electrostatics, vol. 55, no. 2, pp. 173-187, Jun. 2002.

[22] S. Wang, V. Schulz-von der Gathen, and H. F. Dobele, "Discharge comparison of nonequilibrium atmospheric pressure $\mathrm{Ar} / \mathrm{O}_{2}$ and $\mathrm{He} / \mathrm{O}_{2}$ plasma jets," App. Phys. Lett., vol. 83, no. 16, pp. 3272-3274, Oct. 2003.

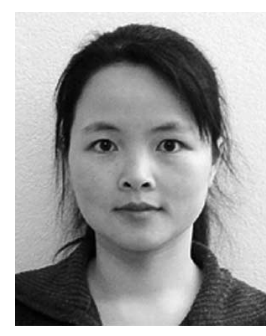

Xutao Deng received the B.Eng. degree in electronic engineering from Shenzhen University, Shenzhen, China, in 1999, the M.Sc.(Eng.) degree in intelligence engineering from the University of Liverpool, Liverpool, U.K., in 2000, and the Ph.D. degree in plasma engineering from Loughborough University, Loughborough, U.K., in 2004.

She is currently a Postdoctoral Research Scientist with the Plasma and Pulsed Power Group, Loughborough University. Her current research interests include protein reduction and bacterial inactivation using atmospheric-pressure glow discharges. 


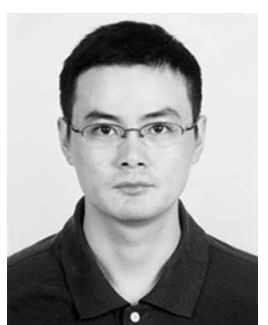

Jianjun Shi received the B.Sc. and M.Sc. degrees in physics from Nanjing University, Nanjing City, China, in 1999 and 2002, respectively, and the Ph.D. degree in electrical engineering from Loughborough University, Leicestershire, U.K., in 2005.

He is currently a Postdoctoral Scientist with the Plasma and Pulsed Power Group, Loughborough University. His research interests include atmospheric-pressure glow discharges and their biomedical applications.

Dr. Shi won an IEEE NSPP Graduate Scholarship Award in 2005.

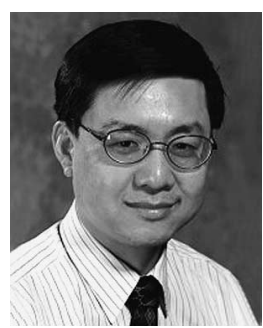

Michael G. Kong (M'94-SM'98) received the B.Sc. and M.Sc. degrees in electronics engineering from Zhejiang University, Hangzhou, China, in 1984 and 1987, respectively, and the Ph.D. degree in electrical engineering from Liverpool University, Liverpool, U.K., in 1992.

After his research and faculty positions with the Liverpool University and Nottingham University, U.K., he joined Loughborough University, Leicestershire, U.K., in 1999, where he holds a Chair in the bioelectrical engineering and leads the Plasma and Pulsed Power Group. At Loughborough University, he is also the Head of the Energy Research Division, whose research encompasses gas plasmas, pulsed power, and renewable energy. He has published approximately 150 papers in scientific journals and peer-reviewed conference proceedings. His current research interests include atmospheric-pressure glow discharges, ultrashort electric pulses, and their biomedical applications.

Dr. Kong is a Guest Editor for a special issue of the IEEE TRANSACTIONS on Plasma SCIENCE on the Nonthermal Medical/Biological Applications of Ionized Gases and Electromagnetic Fields (August 2006). 